\title{
A New Method for Isolating Viable Gonadal Germ Cells from 7-day-old Chick Embryos
}

\author{
Yuki Nakajima $^{1}$, Takeo Minematsu ${ }^{2}$, Mitsuru Naito $^{3}$ and Atsushi Tajima ${ }^{1}$ \\ ${ }^{1}$ Graduate School of Life and Environmental Sciences, University of Tsukuba, Tsukuba, Ibaraki 305-8572, Japan \\ ${ }^{2}$ Department of Advanced Skin Care (Miss Paris), Graduate School of Medicine, The University of Tokyo, \\ Bunkyo-ku, Tokyo 113-0033, Japan \\ ${ }^{3}$ Transgenic Animal Research Center, National Institute of Agrobiological Sciences, Tsukuba, Ibaraki 305-8602, Japan
}

\begin{abstract}
A novel and simple method for isolating viable gonadal germ cells (GGCs) was developed in the domestic chicken, Gallus domesticus. Developing gonads were isolated from 7-day-old chick embryos and cultured for up to 24 hours at $37.8^{\circ} \mathrm{C}$ in phosphate buffered saline without $\mathrm{Ca}^{2+}$ and $\mathrm{Mg}^{2+}$ (PBS [-]). A discharge of GGCs from the gonad was observed within 30 minutes after introducing the embryonic gonad into PBS [-], and the number of discharged GGCs increased until 12 hours of incubation. The purity of the GGCs (number of discharged GGCs/total number of discharged cells) was approximately $50 \%$ for the initial 1.5 hours of incubation and decreased thereafter. The number of discharged GGCs from the left gonad was significantly higher than that from the right gonads in females. Fifty discharged GGCs in PBS [ - ] were injected into the blood stream of 2-day-old chick embryos after staining with PHK26 fluorescent dye. GGCs exhibiting a fluorescent signal were detected after incubating recipient embryos for 5 days.

These results indicate that high-purity, viable GGCs can be collected by simply introducing isolated developing gonads into PBS $[-]$.
\end{abstract}

Key words: chicken, gonadal germ cells, isolation, PBS [-]

J. Poult. Sci., 48: 106-111, 2011

\section{Introduction}

Recent technological progress in producing germline chimeras has provided an important means of studying germline development, genetic resource conservation, and transgenics production. In avian species, primordial germ cells (PGCs) originate from the central zone of the area pellucida (Ginsburg and Eyal-Giladi, 1987; Kagami et al., 1997). At the primitive streak stage, PGCs are found at the extra-embryonic region called the germinal crescent (England and Matsumura, 1993). PGCs circulate temporarily through the bloodstream, followed by migration to the gonadal ridge (Kuwana, 1993; Fujimoto et al., 1976). After migration to the gonadal ridge, PGCs are called gonadal germ cells (GGCs). Gonadal germ cells in the gonadal ridge ultimately differentiate into spermatogonia in the testes or oogonia in the ovaries.

Germline chimeras can be produced in domestic chicken by transferring circulating PGCs (Tajima et al., 1993) or by transferring GGCs collected from the embryonic

Received: September 25, 2010, Accepted: November 18, 2010

Released Online Advance Publication: January 25, 2011

Correspondence: Atsushi Tajima, Graduate School of Life and Environmental Sciences, University of Tsukuba, Tsukuba, Ibaraki 305-

8572, Japan. (E-mail: tajima.atsushi.gb@u.tsukuba.ac.jp) gonad (Tajima et al., 1998; Park et al., 2003; Tajima et al., 2004) into the bloodstream of 2-day-old recipient embryos. GGCs have been isolated commonly by digesting embryonic gonads using proteinases such as trypsin (Tajima et al., 1998). However, it is difficult to purify GGCs from the overwhelming number of somatic cells after digesting the gonad using enzymes.

Therefore, we conducted the present experiments to develop a novel and simple method for isolating GGCs from the gonads of 7-day-old chick embryos.

\section{Materials and Methods}

\section{Experiment 1: Isolation of Gonadal Germ Cells}

Fertilized White Leghorn (WL) and Rhode Island Red (RIR) eggs produced at the Agriculture and Forestry Research Center, University of Tsukuba, Japan were used in the experiments. Fertilized WL eggs were incubated for 7 days in an air-forced incubator (Showa Furanki Laboratory, Saitama, Japan) maintained at $37.8^{\circ} \mathrm{C}$. After incubation, both right and left gonads were isolated from 7 day-old embryos. Isolated gonads were placed in a $1.5 \mathrm{~mL}$ centrifuge tube containing $500 \mu \mathrm{L}$ of phosphate buffered saline without $\mathrm{Mg}^{2+}$ and $\mathrm{Ca}^{2+}$ (PBS [-]). The centrifuge tube was placed in an incubator maintained at $37.8^{\circ} \mathrm{C}$ for $0.5,1,1.5,2,3,4,5,6,9,12,18$, or 24 hours. Cultured 
gonads were pipetted gently, and $100 \mu \mathrm{L}$ of cell suspension was placed on 2-well Heavy Teflon Coated Slides (HTCS, Erie Scientific Co., Portsmouth, NH, USA). The number, survival rate, and purity of discharged GGCs were observed. The purity of discharged GGCs was defined as the ratio between the number of discharged GGCs and the total number of discharged cells expressed as a percentage. The survival rate of isolated GGCs was determined using trypan blue staining. For a control, the same parameters were measured after digesting embryonic gonad using $0.05 \%$ trypsin for 20 minutes in an incubator maintained at $37.8^{\circ} \mathrm{C}$. The morphological characteristics of the GGCs were identical to the PGCs, i.e., large granulated round cells, $14-19 \mu \mathrm{m}$ in diameter with large nuclei (Minematsu et al., 2004), under an inverted microscope (Olympus, Tokyo, Japan). To confirm the histochemical properties of the discharged GGCs, discharged cells were stained with two staining methods, periodic acid-Schiff (PAS) (Tajima et al., 1999) and anti-chicken vasa homologue (CVH) staining, according to the method described by Tsunekawa et al. (2000). For PAS staining, the cell suspension was placed on HTCS, fixed with $10 \%$ formalin in PBS [ - ], and coated with celloidin, and the number of GGCs on each HTCS was determined after the PAS reaction. For CVH staining, the recovered cell suspension was placed on 24-well plates (Falcon, Franklin Lakes, NJ, USA) and fixed with $10 \%$ formalin in PBS $[-]$. After blocking and permeabilization using Blocking One (Nacalai Tesque, Kyoto, Japan), the cells were incubated with rabbit anti-CVH antibodies and treated with alkaline phosphatase-conjugated goat anti-rabbit $\operatorname{IgG}, \operatorname{IgM}$, and IgA (H \& L, 1: 200 dilution; Open Biosystems, Huntsville, AL, USA) as secondary antibodies. Alkaline phosphatase staining was conducted using the BCIP/NBT Substrate System (DakoCytomation, Glostrup, Denmark). The number of GGCs in each plate was assessed after alkaline phosphatase staining. Each experiment was repeated more than five times.

A one-way analysis of variance was conducted using the generalized linear model (GLM; SAS/STAT, SAS Institute Inc., Cary, NC, USA) followed by Tukey's Honestry Significant Difference (HSD) test.

\section{Experiment 2: Sexual Differences in the GGC Numbers from the Right and Left Gonads}

Right and left gonads recovered from 7-day-old embryos were placed separately in a 24-well plate containing $1 \mathrm{~mL}$ PBS [-] and incubated for 30, 60 and 90 minutes. The number of discharged GGCs was counted after CVH staining. A polymerase chain reaction (PCR) was conducted to determinate the gender of the germ cell donors. Each experiment was repeated more than five times.

\section{PCR for Sexing}

Embryonic skin tissue was collected from the leg of each embryo. The sample was placed in DNA extraction buffer (Minematsu et al., 2004), and a $276 \mathrm{bp}$ fragment of the $717 \mathrm{bp} \mathrm{W}$ chromosome-specific XhoI repetitive sequence was amplified according to the methods of Petitte and Kegelmeyer (1995) using the following primer sets:

$5^{\prime}$-CGTGAGAAAAGTGGTAGTT-3' and $3^{\prime}$-CTCTGTCCACCATAAAAACC-5'.

The PCR was conducted with an initial denaturation step at $96^{\circ} \mathrm{C}$ for 2 minutes, followed by 25 cycles at $94^{\circ} \mathrm{C}$ for 30 seconds, $52^{\circ} \mathrm{C}$ for 30 seconds, and $72^{\circ} \mathrm{C}$ for 30 seconds, with a final extension step at $72^{\circ} \mathrm{C}$ for 5 minutes in a Takara PCR Thermal Cycler SP (TP-400; Takara Shuzo, Kyoto, Japan). The PCR products were separated by electrophoresis on a 1.5\% agarose gel and visualized under UV light after staining with ethidium bromide. Only samples that were obtained from females were expected to express the $276 \mathrm{bp}$ PCR product.

A split-plot design was used to conduct the experiment, and the data were analyzed using the GLM procedure, followed by Tukey's HSD test using SAS/STAT.

\section{Experiment 3: Migratory Ability of the GGCs}

Right and left gonads from each embryos were placed in $1.5 \mathrm{~mL}$ centrifuge tubes and incubated in $100 \mu \mathrm{L} \mathrm{PBS} \mathrm{[-]}$ for 1 hour. Discharged GGCs were labeled with PKH26 fluorescent dye (Zynaxis, Inc., Malvern, PA, USA) and 50 GGCs were injected into the dorsal aorta of RIR embryos at stages 13-16 of development (Hamburger and Hamilton, 1951) using a fine glass pipette under a dissection microscope. Recipient eggs were prepared 1 hour before injection to remove approximately $5 \mu \mathrm{L}$ of blood through a small window (about $1 \mathrm{~cm}$ diameter) in the eggshell using a fine glass pipette under a dissection microscope. After the injection, the window was sealed with plastic tape, and the egg was incubated at $37.8^{\circ} \mathrm{C}$ for 5 days. Right and left gonads were collected and placed in $1.5 \mathrm{~mL}$ centrifuge tubes containing $50 \mu \mathrm{L}$ of $0.05 \%$ trypsin in PBS [- ] and incubated at $37.8^{\circ} \mathrm{C}$ for 20 minutes. After the incubation, the cells were placed on HTCS, and the total number of fluorescently labeled cells recovered from each gonad were counted under a fluorescence microscope (IMT-2, Olympus, Tokyo, Japan) using a 546-nm excitation filter.

All animal care and handling procedures were performed in accordance with the standards of the University of Tsukuba.

\section{Results}

A discharge of GGCs from gonad of 7-day-old chick embryos after a 1-hour incubation in PBS [- ] at $37.8^{\circ} \mathrm{C}$ was shown in Fig. 1 and discharged cells stained with either PAS or anti-CVH was shown in Fig. 2. The proportion of PAS positive morphological GGCs was $95 \%$ until 8 hours after the introduction of the gonads into PBS [-], and the proportion decreased to $90 \%$ at 24 hours (data not shown). The proportion of anti-CVH-positive morphological GGCs was $>93 \%$ at 24 hours of incubation (data not shown).

The temporal change in the number, survival rate, and purity of GGCs discharged from incubated embryonic gonads in PBS [-] during 24 hours is shown in Fig. 3. The number of discharged GGCs at 30 minutes and 12 
hours after incubation were $402 \pm 55.6$ and $2,438 \pm 190.4$, respectively $(P<0.05)$. However, no further increase in the number of discharged GGCs was observed between 12 and 24 hours of incubation. The GGC purity, defined as the proportion of GGCs vs. the total number of observed cells, at $0.5,1$, and 1.5 hours after the incubation was 46.9 $\pm 5.9 \%, 48.2 \pm 6.3 \%$, and $51.3 \pm 6.6 \%$, respectively $(P>$ $0.05)$, but the value decreased significantly thereafter and reached $20 \%$ at 24 hours. For comparison, the number of dissociated GGCs and the GGC purity were 1,052 \pm 239 and $4.26 \pm 0.49 \%$, respectively, when trypsin was used to dissociate the gonadal tissue isolated from 7-day-old chick embryos. The survival rate of discharged GGCs remained
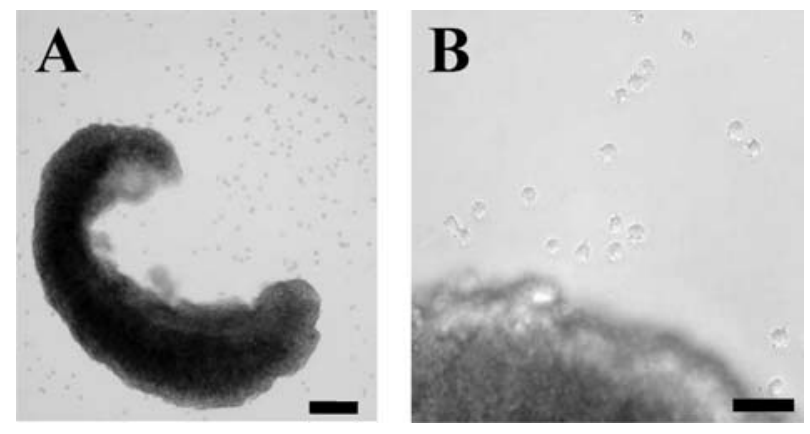

Fig. 1. Discharge of gonadal germ cells (GGCs) from chick embryonic gonads after a 1-hour incubation in phosphate-buffered saline without $\mathrm{Ca}^{2+}$ and $\mathrm{Mg}^{2+}$ (PBS [-]) at $37.8^{\circ} \mathrm{C}$. (A) Gonad of 7-day-old chick embryos. $\mathrm{Bar}=200 \mu \mathrm{m}$ (B) $\mathrm{Bar}=50 \mu \mathrm{m}$.
$>80 \%$ for the first 9 hours of gonadal incubation $(P>$ $0.05)$, followed by a significant decrease thereafter, and reached $40 \%$ at 24 hours $(P<0.05)$.
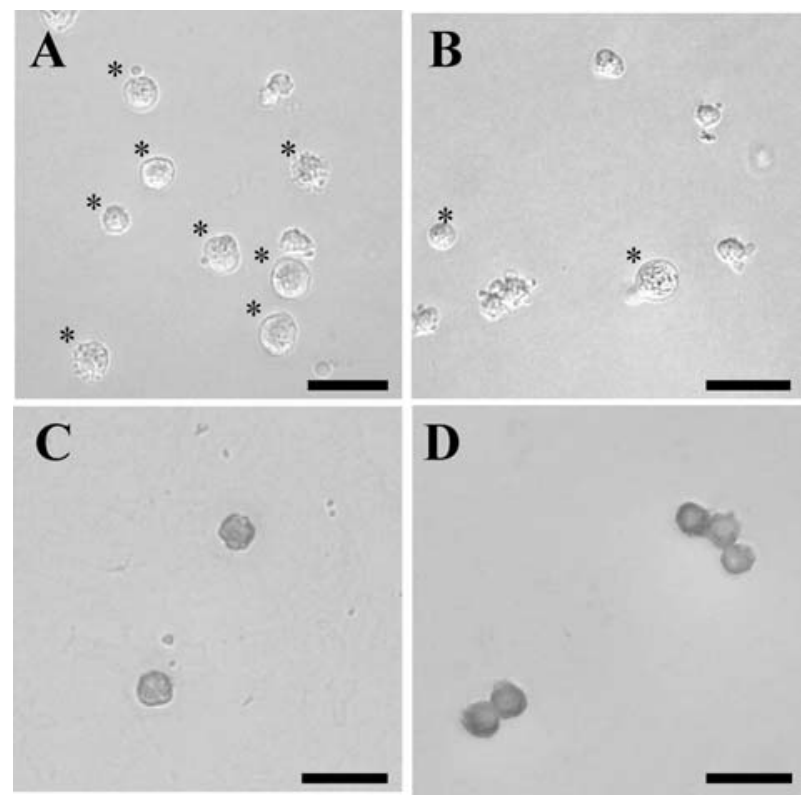

Fig. 2. Discharged cells after incubating 7-day-old chick embryos in (A) phosphate-buffered saline without $\mathrm{Ca}^{2+}$ and $\mathrm{Mg}^{2+}$ (PBS $[-]$ ) for 1 hour, (B) trypsin treatment, (C) periodic acid-Schiff (PAS) staining, and (D) antichicken vasa homologue (anti-CVH) staining. *: gonadal germ cells (GGCs), Bar $=30 \mu \mathrm{m}$.

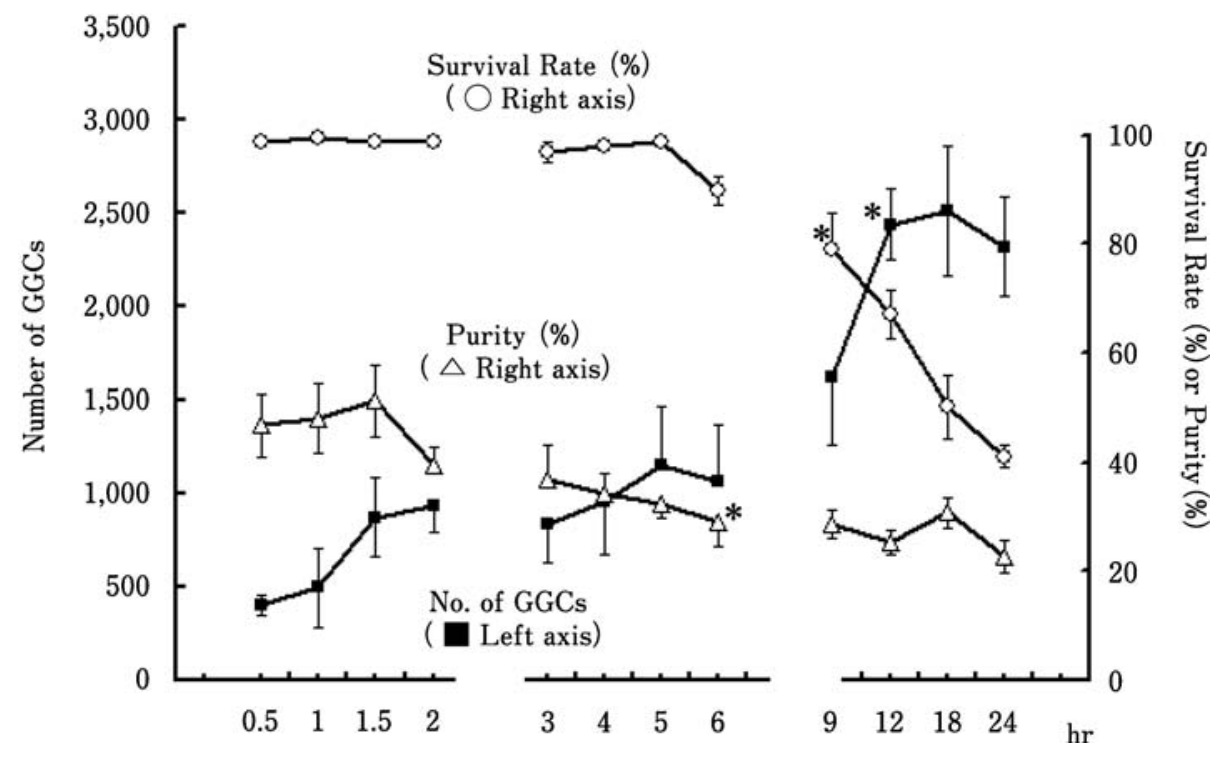

Fig. 3. Number, survival rate and purity of gonadal germ cells (GGCs) discharged from embryonic gonad. *: First observation of significant difference compared with the value at 30 minutes after incubation $(P<0.05)$ 


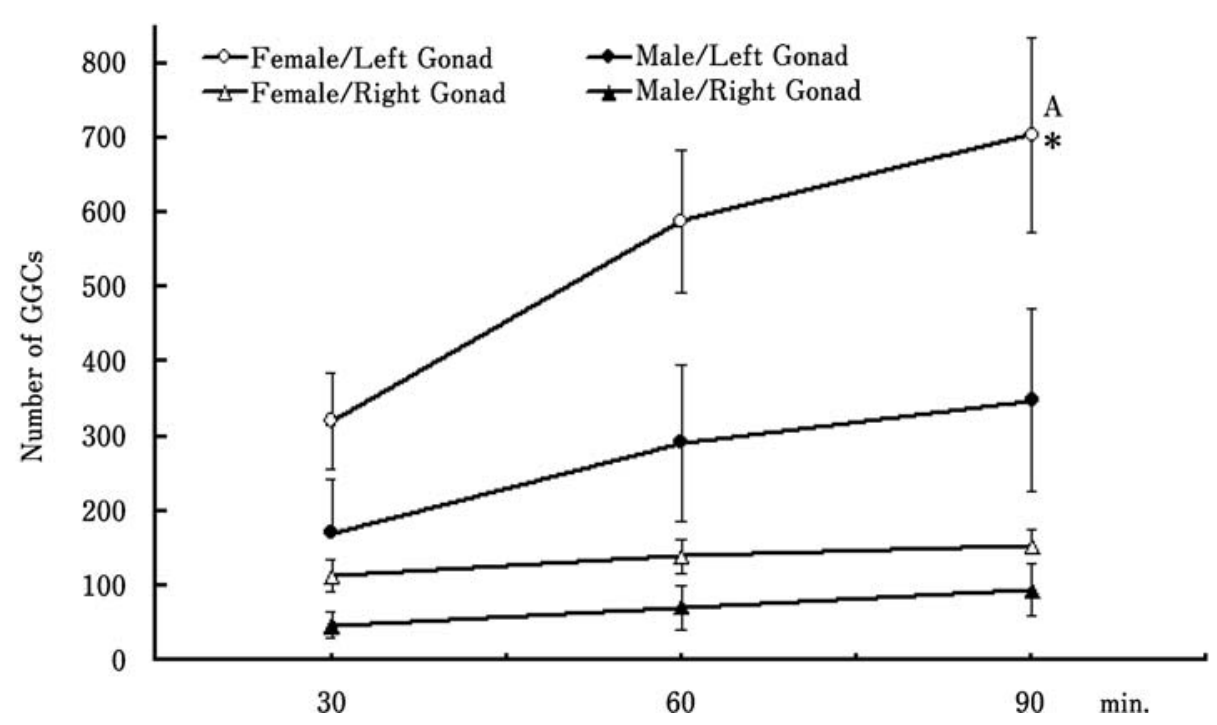

Fig. 4. Sexual differences in the number of discharged gonadal germ cells (GGCs) after incubating right and left embryonic gonads in phosphate-buffered saline without $\mathrm{Ca}^{2+}$ and $\mathrm{Mg}^{2+}$ (PBS $[-])$. A: Significantly different from other treatments within each incubation time $(P<0.05)$ *: First observation of significant difference compared with the value at 30 minutes after incubation $(P<0.05)$.

The number of GGCs discharged into PBS [-] from the right and left gonad by gender of the embryo is shown in Fig. 4. In both male and female gonads, the number of GGCs discharged into PBS [-] after the incubation was significantly higher from the left gonad than from the right $(P<0.05)$.

The migratory ability of the GGCs collected by the PBS [-] method is shown in Table 1. When 50 fluorescently labeled GGCs were transferred into recipient embryos, $78.7 \pm 11.5$ fluorescently labeled GGCs were detected, among which $45.2 \pm 5.3$ and $33.6 \pm 7.3$ were detected from the left and right gonads, respectively $(P<0.05)$.

\section{Discussion}

In the present study, highly pure (approximately 50\% of total cells) viable GGCs that retained migratory ability toward the developing gonad were recovered efficiently by simply culturing embryonic gonads in PBS $[-]$ at $37.8^{\circ} \mathrm{C}$ for 0.5-1.5 hours (Fig. 3).

Although the underlying mechanism for the predominant discharge of GGCs from the developing gonad into PBS [ - ] for the first 1.5 hours after the introduction of embryonic gonad is not understood at present, this rapid and simple method of isolating GGCs from developing gonads will considerably facilitate the study of germline chimera production. The predominant initial discharge of GGCs into the medium may indicate a weak cellular association between discharged GGCs and gonadal somatic cells in the embryonic gonad of 7-day-old chick embryos.
A significant increase in the number of discharged GGCs was observed between 9 and 12 hours after introducing the embryonic gonads into PBS [-] (Fig. 3). However, the GGC purity did not change during the same period. It was speculated from these results that spontaneous gonadal dissociation was accelerated by 9 hours after incubating the embryonic gonads in PBS [-]. Alternatively, the present result may indicate a depletion of unassociated free GGCs in the embryonic gonad. The significant decrease in the survival rate of GGCs over 9 hours of incubation could be attributed to depletion of nutrients essential for maintaining the cellular metabolism of discharged GGCs.

Although a morphological analysis of embryonic gonads has been reported (Drews et al., 1988; Civinini et al., 1994), few reports are available on the cellular association between germ cells and somatic cells in the gonads. It is generally accepted that the gonadal somatic cells support maturation and differentiation of germ cells in the gonad (Johnson, 2000; Kirby and Froman, 2000). Further study should be conducted to understand the development and differentiation of germ cells in relation to the mode of cellular association between GGCs and gonadal somatic cells.

It was observed that the GGCs were released mainly from the edge of the gonad where the gonad was previously attached to the kidney (Fig. 1). It was, therefore, hypothesized that the developing medullary cords in male or cortical cords in females may play a role in the discharge of GGCs. 
Table 1. Number of PKH26 positive gonadal germ cells (GGCs) in the gonad of recipient embryos that were transferred 50 GGCs. $(\mathbf{N}=38)$

\begin{tabular}{lccc}
\hline \hline & Left Gonad $^{1)}$ & Right Gonad $^{1)}$ & Total \\
\hline $\begin{array}{l}\text { The number of PKH26 } \\
\text { positive GGCs }\end{array}$ & $45.2 \pm 5.3$ & $33.6 \pm 7.3$ & $78.7 \pm 11.5$ \\
\hline $\begin{array}{l}\text { 1) } \\
\text { The number of PKH26 positive GGCs differs significantly between right and left } \\
\text { gonad using a paired t-test }(P<0.05) .\end{array}$ & &
\end{tabular}

A histological study to compare the differences in gonadal structure before and after incubation in PBS $[-]$ needs to be conducted to elucidate the GGC discharge mechanism.

Furthermore, the chemical and physical factors affecting GGC discharge into the medium, i.e., medium composition, incubation temperature, and gas environments need to be studied.

The concentration of circulating PGCs in the blood is highest at either stage 14 or 15 in chick embryos (Tajima et al., 1999). However, the incubation time required to reach developmental stages 14-15 varies considerably among individual embryos (Tajima et al., 1999), and this can only be observed after exposing the embryo from the egg shell. Furthermore, the proportion of PGCs against blood cells is reported to be $0.02 \%$ (Yasuda et al., 1992). Therefore, multiple eggs must be sacrificed to ensure the collection of circulating PGCs from embryos at stages 1415 to produce germline chimeras. The scarcity of circulating PGCs has been a major limiting factor in research on germline development. In this respect, the method of collecting viable GGCs from developing gonads, as described in the present study, has a clear advantage of recovering viable germ cells when the availability of fertilized embryos is limited, such as the case with lowfertility strains or wild birds.

It was observed that the intensity of PAS staining against GGCs collected from 7-day-old embryos was weaker after a 24-hour incubation than with GGCs collected from embryos incubated for less than 24 hours (data not shown). Because the Schiff reagent used in PAS staining reacts with the aldehyde residue produced by treating samples with periodic acid, which dissociates the covalent bonds between the second and third carbon residue of the glycopyranose, it was speculated that there was a depletion of glycogen in the cytoplasm of the GGCs.

A significantly higher number of GGCs was discharged from the left gonad in females in this study (Fig. 4). The same tendency was observed in male gonads, although no statistically significant difference was observed. This observation might simply be due to an asymmetrical migration of PGCs in favor of the left gonad over the right gonad, which has been reported previously (Zaccanti et al., 1990; Nakamura et al., 2007; Naito et al., 2009). A1ternatively, the post-migratory proliferation of GGCs may have been higher in the left than in the right gonad. Reasons for the higher number of discharged GGCs from the left gonad of females need to be studied.

Fluorescently labeled GGCs were found in gonads from recipient embryos using the method of discharging the GGCs into PBS [-], indicating that the GGCs discharged into PBS [-] retained their migratory ability in the developing gonad (Table. 1). A progeny test should be conducted by transferring GGCs collected with the PBS [-] method.

Seven-day-old chick embryos were used in the present study. Although germline chimeras have been produced by transferring GGCs collected from 5-day (Tajima et al., 1998) as well as 7- and 9-day-old embryos (Tajima et al., 2004), the biological nature of GGCs is currently not fully understood. Future study should be directed toward extending the present method to the entire period of embryonic development.

In conclusion, viable GGCs were collected by simply culturing 7-day-old gonads from chick embryos. The results provide an alternative means for producing germline chimeras to conserve genetic resources and to produce transgenic avian species. Future experiments should examine the discharge of GGCs from other than 7-day-old embryos.

\section{Acknowledgments}

The authors acknowledge the staff of the Agricultural and Forestry Research Center, University of Tsukuba, for their technical support in maintaining the animals. This study was supported in part by a Grant-in-Aid (No. 20380156) from the Japan Society for the Promotion of Science to MN.

\section{References}

Civinini A, Gallo VP and Petrucci S. Chick gonadogenesis following early surgical bursectomy. II: Ultrastructural data on the embryonic left and right female gonads. Anatomy and Embryology, 190: 439-444. 1994.

Drews U, Ebensperger $\mathrm{C}$ and Wolf $\mathrm{U}$. An in vitro model of gonad differentiation in the chick embryo. Roller cultures in gas permeable biofoil bags. Anatomy and Embryology, 178: 529-536. 1988.

England MA and Matsumura G. Primordial germ cells in the primitive streak stages chick embryo as studied by scanning electron microscopy. Journal of Anatomy, 183: 67-73. 1993.

Fujimoto T, Ukeshima A and Kiyofuji R. The origin, migration and morphology of the primordial germ cells in the chick embryo. Anatomical Record, 185: 139-153. 1976.

Ginsburg $\mathbf{M}$ and Eyal-Giladi H. Primordial germ cells of the 
young chick blastoderm originate from the central zone of the area pellucida irrespective of the embryo-forming process. Development, 101: 209-219. 1987.

Hamburger V and Hamilton HL. A series of normal stages in the development of the chick embryo. Journal of Morphology, 88: 49-92. 1951.

Johnson AL. Reproduction in the female. In: Sturkie's Avian Physiology (Whittow GC eds.). $5^{\text {th }}$ ed. pp. 569-596. Academic Press. London. 2000.

Kagami H, Tagami T, Matsubara Y, Harumi T, Hanada H, Maruyama K, Sakurai M, Kuwana T, and Naito M. The developmental origin of primordial germ cells and the transmission of the donor-derived gametes in mixed-sex germline chimeras to the offspring in the chicken. Molecular Reproduction and Development, 48: 501-510. 1997.

Kirby JD and Froman DP. Reproduction in the male. In: Sturkie's Avian Physiology (Whittow GC eds.). $5^{\text {th }}$ ed. pp. 597-616. Academic Press. London. 2000.

Kuwana T. Migration of avian primordial germ cells toward the gonadal anlage. Development Growth and Differentiation, 35: 237-243. 1993.

Minematsu T, Tajima A and Kanai Y. The migratory ability of gonadal germ cells in the domestic chicken. Journal of Poultry Science, 41: 178-185. 2004.

Naito M, Minematsu T, Harumi T and Kuwana T. Preferential migration of transferred primordial germ cells to left germinal ridge of recipient embryos in chickens. Journal of Poultry Science, 46: 40-45. 2009.

Nakamura Y, Yamamoto Y, Usui F, Mushika T, Ono T, Setioko R, Takeda K, Nirasawa K, Kagami $H$ and Tagami $T$. Migration and proliferation of primordial germ cells in early chicken embryo. Poultry Science, 86: 2182-2193. 2007.

Park TS, Jeong DK, Kim JN, Song GH, Hong YH, Lim JM and Han JY. Improved germline transmission in chicken chime- ras produced by transplantation of gonadal primordial germ cells into recipient embryos. Biology of Reproduction, 68: 1657-1662. 2003.

Petitte JN and Kegelmeyer AE. Rapid sex determination of chick embryos using the polymerase chain reaction. Animal Biotechnology, 6: 119-130. 1995.

Tajima A, Naito M, Yasuda Y and Kuwana T. Production of germ line chimera by transfer of primordial germ cells in the domestic chicken (Gallus domesticus). Theriogenology, 40: 509-519. 1993.

Tajima A, Naito M, Yasuda Y and Kuwana T. Production of germ-line chimeras by transfer of cryopreserved gonadal primordial germ cells (gPGCs) in chicken. Journal of Experimental Zoology, 280: 265-267. 1998.

Tajima A, Hayashi H, Kamizumi A, Ogura J, Kuwana T and Chikamune T. Study on the concentration of circulating primordial germ cells (cPGCs) in early chick embryos. Journal of Experimental Zoology, 284: 759-764. 1999.

Tajima A, Minematsu T and Ohara M. Production of germ-line chimeras by the transfer of cryopreserved gonadal germ cells collected from 7- and 9-day-old chick embryos. Animal Science Journal, 75: 85-88. 2004.

Tsunekawa N, Naito M, Sakai Y, Nishida T and Noce T. Isolation of chicken vasa homolog gene and tracing the origin of primordial germ cells. Development, 127: 2741-2750. 2000.

Yasuda Y, Tajima A, Fujimoto T and Kuwana T. A method to obtain avian germ-line chimeras using isolated primordial germ cells. Journal of Reproduction and Fertility, 96: 521528. 1992.

Zaccanti F, Vallisneri M and Quaglia A. Early aspects of sex differentiation in the gonads of chick embryos. Differentiation, 43: 71-80. 1990. 